\title{
Integration of BOLD-fMRI and DTI into radiation treatment planning for high-grade gliomas located near the primary motor cortexes and corticospinal tracts
}

Minglei Wang ${ }^{1 \dagger}$, Hui Ma ${ }^{2,3}$, Xiaodong Wang ${ }^{1,2^{*}+}$, Yanhong Guo ${ }^{4}$, Xinshe Xia ${ }^{4}$, Hechun Xia ${ }^{2,3}$, Yulin Guo ${ }^{1}$, Xueying Huang ${ }^{1}$, Hong $\mathrm{He}^{5}$, Xiaoxiong $\mathrm{Jia}^{3}$ and Yan Xie

\begin{abstract}
Background: The main objective of this study was to evaluate the efficacy of integrating the blood oxygen level dependent functional magnetic resonance imaging (BOLD-fMRI) and diffusion tensor imaging (DTI) data into radiation treatment planning for high-grade gliomas located near the primary motor cortexes (PMCs) and corticospinal tracts (CSTs).

Methods: A total of 20 patients with high-grade gliomas adjacent to PMCs and CSTs between 2012 and 2014 were recruited. The bilateral PMCs and CSTs were located in the normal regions without any overlapping with target volume of the lesions. BOLD-fMRI, DTI and conventional MRI were performed on patients (Karnofsky performance score $\geq 70$ ) before radical radiotherapy treatment. Four different imaging studies were conducted in each patient: a planning computed tomography (CT), an anatomical MRI, a DTI and a BOLD-fMRI. For each case, three treatment plans (3DCRT, IMRT and IMRT_PMC\&CST) were developed by 3 different physicists using the Pinnacle planning system.

Results: Our study has shown that there was no significant difference between the 3DCRT and IMRT plans in terms of dose homogeneity, but IMRT displayed better planning target volume (PTV) dose conformity. In addition, we have found that the Dmax and Dmean to the ipsilateral and contralateral PMC and CST regions were considerably decreased in IMRT_PMC\&CST group $(p<0.001)$.

Conclusions: In conclusion, integration of BOLD-fMRI and DTI into radiation treatment planning is feasible and beneficial. With the assistance of the above-described techniques, the bilateral PMCs and CSTs adjacent to the target volume could be clearly marked as OARs and spared during treatment.

Keywords: High-grade gliomas, Blood oxygen level dependent functional magnetic resonance imaging (BOLD-fMRI), Diffusion tensor imaging (DTI), Three-dimensional conformal radiation treatment (3DCRT), Intensity-modulated radiation therapy (IMRT), Radiation treatment planning
\end{abstract}

\section{Background}

Gliomas, which contain oligodendroglia, astrocytic and ependymal lesions are the most common primary intracranial tumors. High-grade gliomas, which make up 35 to $45 \%$ of all newly diagnosed primary brain tumors worldwide, have a very poor prognosis [1]. The three-dimensional

\footnotetext{
* Correspondence: xdw80@yeah.net

${ }^{\dagger}$ Equal contributors

'Department of Radiology, General Hospital of Ningxia Medical University, Yinchuan, China

${ }^{2}$ Ningxia Key Laboratory for Cerebrocranial Diseases, Yinchuan, China Full list of author information is available at the end of the article
}

conformal radiotherapy (3DCRT) has been considered as the standard therapy for patients with high-grade gliomas and intensity-modulated radiotherapy (IMRT) is becoming increasingly used to improve dose conformity and spare critical normal tissues. However, the risk of radiationinduced brain injury increases with the increase of radiation dose [2-4]. The strenuous endeavor has been made to diminish radiation complications.

With the assistance of conventional magnetic resonance imaging (MRI) and planning computed tomography (CT) data, many critical intracranial structures, such as lens,

\section{Ciomed Central}

(c) 2015 Wang et al.; licensee BioMed Central. This is an Open Access article distributed under the terms of the Creative Commons Attribution License (http://creativecommons.org/licenses/by/4.0), which permits unrestricted use, distribution, and reproduction in any medium, provided the original work is properly credited. The Creative Commons Public Domain Dedication waiver (http://creativecommons.org/publicdomain/zero/1.0/) applies to the data made available in this article unless otherwise stated. 
optic nerves and optic chiasm are well demarcated. However, it is difficult to accurately locate eloquent cortices and fiber connections in the white matter of the brain by routine neuroimaging. Excessive irradiation of eloquent cortices and white matter fiber tracts is unavoidable. Blood oxygen level dependent functional magnetic resonance imaging (BOLD-fMRI) and diffusion tensor imaging (DTI) have recently been used to identify the primary motor cortexes (PMCs) and corticospinal tracts (CSTs). These imaging techniques have been implemented in modern neuronavigation systems and used to guide the surgical removal of critically located intracranial lesions $[5,6]$. The purpose of our study was to evaluate whether the incorporation of BOLD-fMRI and DTI data into the 3D treatment planning process could spare the healthy brain and sensitive parts of the brain from high doses of radiation.

\section{Methods}

Ethical Approval was obtained from the General Hospital of Ningxia Medical University Review Board and written informed consent was obtained from patients. The study was conducted with strict adherence to the Declaration of Helsinki Principles.

\section{Subjects}

A total of 20 patients with high-grade gliomas adjacent to PMCs and CSTs between May 2012 and February 2014 were recruited from the General Hospital of Ningxia Medical University, China. Eleven male patients and 9 female patients aged from 24 to 66 year-old were enrolled in this study.

The glioma tissues in our study included 14 astrocytomas (WHO Grade III) and 6 glioblastomas (WHO Grade IV). The bilateral PMCs and CSTs were located in the normal regions without any overlapping with target volume of the lesions. BOLD-fMRI, DTI and conventional MRI were performed on patients (Karnofsky performance score $\geq 70$ ) before radical radiotherapy treatment.

\section{Data acquisition and analysis}

Four different imaging studies were conducted in each patient: a planning CT for radiosurgery treatment and target tracking during radiation therapy treatment delivering; an anatomical MRI to deliver a complete set of morphological MR data; a DTI to provide white matter tractography and a BOLD- fMRI to provide brain activation maps. Axial CT images (3-mm slice thickness) were taken by a wide-bore Siemens Somatom Sensation Open CT scanner (Siemens, Germany). MRI volumes were acquired using a Signal HDx 3.0 T MRI scanner (General Electric Company, USA).

BOLD fMRI data were obtained using fat-saturated single-shot gradient echo planar imaging $(\mathrm{EPI})(\mathrm{TE}=35 \mathrm{~ms}$, $\mathrm{TR}=3,000 \mathrm{~ms}$, acquisition matrix $=64 \times 64$ pixels, $\mathrm{FOV}=$
$240 \mathrm{~mm} \times 240 \mathrm{~mm}^{2}$, flip angle $=90^{\circ}, \mathrm{NEX}=1,3 \mathrm{~mm}$ thickness). A block design paradigm (5 cycles, $30 \mathrm{sec}$ on and $30 \mathrm{sec}$ off) was utilized. Functional areas relevant to each treatment region were probed by Somatosensory tasks (finger tapping with audio cue). Following the acquisition of the functional data, gadolinium-enhanced high-resolution images were acquired $\left(\mathrm{TR} / \mathrm{TE}=450 / 14\right.$, flip angle $=90^{\circ}$, matrix $=256 \times 256$, FOV $=240 \mathrm{~mm} \times 240 \mathrm{~mm}^{2}$, and slice thickness $3 \mathrm{~mm}$ skip $0 \mathrm{~mm}$ ). After the images were taken, data were transferred to the Matlab workstation for analysis. The DTI data acquisition sequence was a spin echo-echo planar imaging (SE-EPI) sequence with $\mathrm{TR}=10,000 \mathrm{~ms}$, $\mathrm{TE}=98.8 \mathrm{~ms}$, acquisition matrix $=128 \times 128$ pixels; FOV $=$ $240 \mathrm{~mm} \times 240 \mathrm{~mm}^{2}$; slice thickness $=3.0 \mathrm{~mm}$. Diffusionweighted imaging with $\mathrm{b}$ factor of $1,000 \mathrm{~mm}^{2} / \mathrm{s}$ was taken along 25 noncollinear directions. The acquisition time of DTI sequence was 280 seconds. DTI data was analyzed online by the advantage workstation of the MR scanner (AW 4.4). For CST analysis, a seed region of interest (ROI) and a target ROI were placed on the posterior limb of the internal capsule and pons (anterior blue portion on the color map). Fiber tracking employed fractional anisotropy (FA) threshold of 0.2 and a tract angular change of $30^{\circ}$. The color-coded FA maps were merged with the anatomical MRI images. Regions of interest were drawn on the fused FA maps.

The fused fMRI activation maps and the white matter tracts overlaid on the anatomical MRI volume were exported as separate grayscale dicom images and loaded onto Pinnacle planning system software version 9.2 (Philips Medical Systems, Netherlands). The anatomical MRI images were registered with the CT volume for each patient. Figure 1 shows the anatomical MRI images were

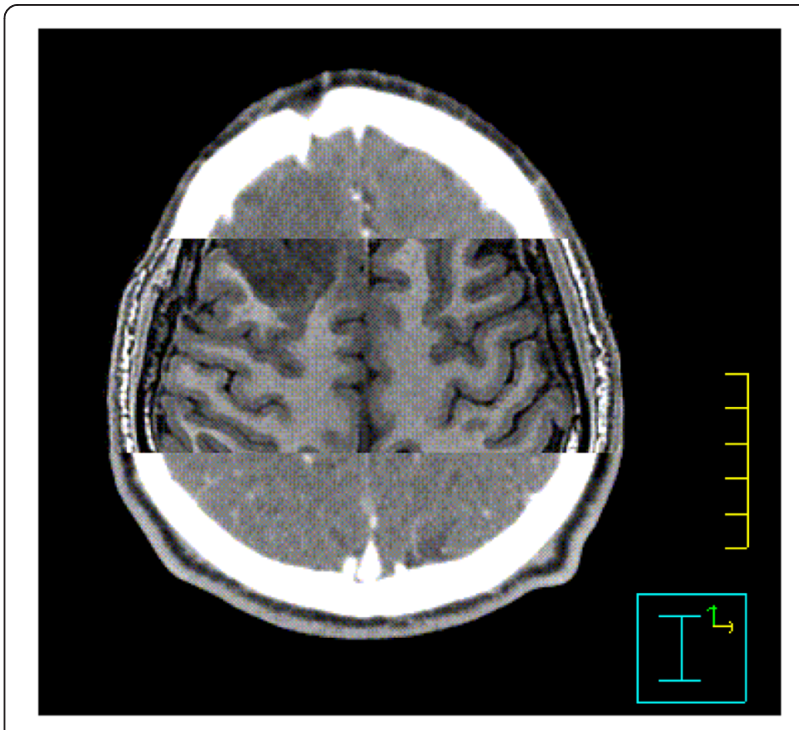

Figure 1 T1-weighted MR imaging and the corresponding axial $\mathrm{CT}$ after registration. 
registered with the corresponding axial CT planes for a glioma case.

\section{Treatment planning}

The target and organs at risk (OARs), i.e., optic nerves, optic chiasm and brain stem were precisely described using CT/anatomical MRI images. Both eyes were protected to avoid beam damage during treatment planning. The PMCs and the CSTs situated near the target were defined by a radiologist and a neurosurgeon, using the tractography images and the fused activation maps. Gross tumor volume
(GTV) was described as the operative cavity with any remaining contrast-enhancing tissue on T1-weighted magnetic resonance imaging or as unresected enhancing tumor. The initial clinical target volume (CTV1) was defined as the T2 hyper intensity area (edema) with a $20 \mathrm{~mm}$ expansion. An initial planning target volume (PTV1) was created by adding a $30 \mathrm{~mm}$ expansion to the CTV1 to account for setup uncertainties. A second clinical target volume (CTV2) was defined as the contrast enhancement region in T1 with an additional $25 \mathrm{~mm}$ margin. A PTV2 was generated by adding a $30 \mathrm{~mm}$ expansion to the CTV2.
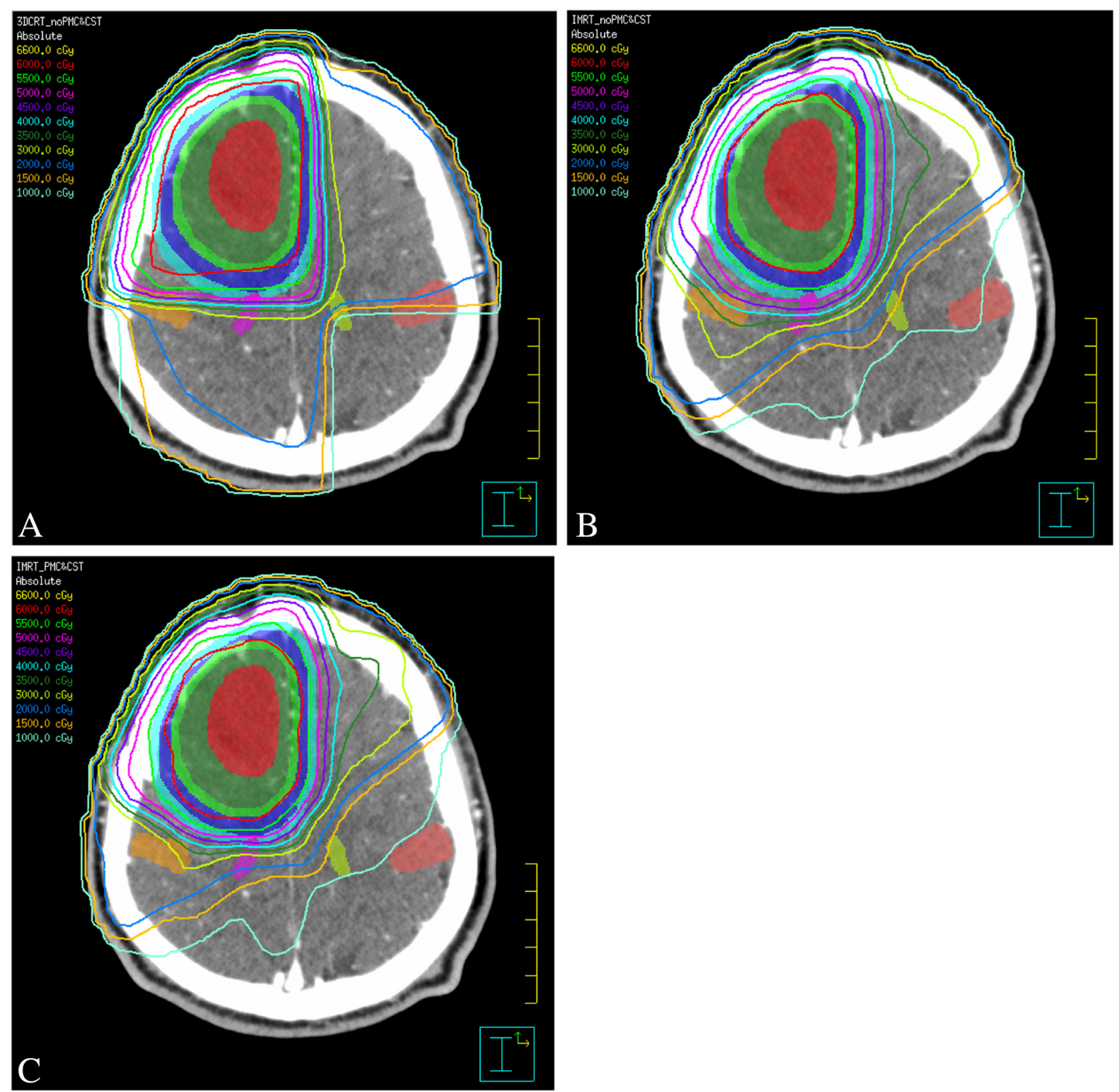

Figure 2 Axial isodose distribution in a patient with high-grade glioma. The $\mathbf{A}, \mathbf{B}$ and $\mathbf{C}$ show the dose distributions for 3DCRT, IMRT and IMRT_PMC\&CST, respectively. (orange) ipsilateral PMC (red square symbol) contralateral PMC (pink square symbol) ipsilateral CST (green square symbol) contralateral CST. 
For each case, three treatment plans were developed by 3 different physicists using the Pinnacle planning system. The first physicist created conventional 3DCRT plans (3DCRT) and the PMCs and the CSTs situated near the target were not taken in account by the physicist. The target and standard morphological OARs were considered in this plan. The second physicist developed IMRT plans (IMRT) and the PMCs and the CSTs situated near the target were not considered by the physicist. The third physicist developed IMRT plans (IMRT_PMC\&CST), and the PMCs and the CSTs situated near the target were considered. Figure 2 shows the axial isodose distribution of a patient with high-grade glioma.

The treatment plans met the requirement that at least 95\% of the PTV receives the prescribed dose. Cumulative doses to the lenses, optic nerves, optic chiasm, and brainstem were limited to a maximum dose of 54 Gy for the last three structures and as low as practically achievable for the former. For conventional 3DCRT treatment, the prescribed dose was 50 Gy to the PTV1, immediately followed by 10 Gy to the PTV2, with a total cumulative dose of 50 Gy to the PTV1 and 60 Gy to the PTV 2 both at 2 Gy per fraction. For IMRT plans, the prescribed dose was 50 Gy to the PTV1 and 60 Gy to the PTV2, which were delivered concurrently over 30 daily fractions, with a fractional dose of 2 Gy to the PTV2.

\section{Comparison criteria for the radiation treatment plans}

The dose volume histograms (DVH) data were obtained from each patient. The dose coverage was analyzed according to the mean dose (Dmean), maximum dose (Dmax), conformity index (CI) and homogeneity index (HI). The CI was defined as follows [7]: $\mathrm{CI}=\mathrm{VRI} / \mathrm{PTV}$, where VRI represents the volume covered by the prescription dose. A CI value of 1.0 indicates that the volume of the prescription isodose surface is equal to that of the PTV. The HI was defined as follows [8]: $\mathrm{HI}=(\mathrm{D} 2-\mathrm{D} 98) / \mathrm{D} 50$, where Dx\% represents the dose delivered to $\mathrm{x} \%$ of the PTV. Lower HI values indicate a more homogeneous target dose. OARs (e.g., brainstem, optic chiasm, optic nerves, and lenses) and PMCs and CSTs were compared based on the values of Dmax and Dmean.

\section{Statistical analyses}

The comparison of parameters between different plans was analyzed by the paired two-tailed Student $t$ test. Differences were considered statistically significant at $p<0.05$.

\section{Results}

Comparison of target volume coverage and OAR sparing between 3DCRT and IMRT

Parameters related to dose coverage planning for 3DCRT and IMRT are presented in Tables 1 and 2. The results indicated that there was no significant difference between the 3DCRT and IMRT plans in terms of dose
Table 1 Comparison of target volume coverage between 3DCRT and IMRT

\begin{tabular}{lllll}
\hline & 3DCRT & IMRT & $\boldsymbol{t}$ & $\boldsymbol{p}$ \\
\hline PTV1 (50 Gy) & & & & \\
$\mathrm{D}_{\max }$ (Gy) & $65.06 \pm 0.46$ & $64.88 \pm 0.66$ & 1.461 & 0.160 \\
$\mathrm{D}_{\text {mean }}$ (Gy) & $59.78 \pm 0.77$ & $59.91 \pm 0.85$ & -0.551 & 0.588 \\
Cl & $1.219 \pm 0.054$ & $1.071 \pm 0.025$ & 10.492 & $<0.001^{*}$ \\
HI & $0.210 \pm 0.008$ & $0.213 \pm 0.012$ & -1.177 & 0.254 \\
PTV2 (60 Gy) & & & & \\
$D_{\text {max }}$ (Gy) & $65.06 \pm 0.46$ & $64.88 \pm 0.66$ & 1.461 & 0.160 \\
$D_{\text {mean }}$ (Gy) & $62.46 \pm 0.39$ & $62.36 \pm 0.53$ & 1.301 & 0.209 \\
Cl & $1.178 \pm 0.082$ & $1.055 \pm 0.049$ & 5.552 & $<0.001^{*}$ \\
HI & $0.086 \pm 0.022$ & $0.082 \pm 0.016$ & 0.809 & 0.429 \\
\hline *Significant difference. & & &
\end{tabular}

homogeneity, but IMRT displayed better PTV dose conformity. Regarding the comparison of PTV1 Dmax, PTV1 Dmean, PTV2 Dmax and PTV2 Dmean, there was no significant difference between the 3DCRT and IMRT plans. The dosimetric details of brainstem, optic chiasm, optic nerves, and lenses revealed no significant differences between the two plans and all of these organs were strictly maintained within the dose limitations. The Dmax and Dmean of PMCs and CSTs were observed in both 3DCRT and IMRT plans (Table 3); however no significant difference was found between the two plans.

\section{Comparison of target volume coverage and OAR sparing between IMRT and IMRT_PMC\&CST}

Treatment plan parameters are shown in Tables 4 and 5 . According to the data presented, both PTV1 and PTV2

Table 2 Comparison of OAR sparing between 3DCRT and IMRT

\begin{tabular}{|c|c|c|c|c|}
\hline & 3DCRT (Gy) & IMRT (Gy) & $t$ & $p$ \\
\hline Ipsilateral lens $D_{\max }$ & $1.99 \pm 1.00$ & $2.67 \pm 1.67$ & -1.938 & 0.068 \\
\hline Ipsilateral lens $D_{\text {mean }}$ & $1.52 \pm 0.75$ & $1.98 \pm 1.45$ & -1.454 & 0.162 \\
\hline Contralateral lens $D_{\max }$ & $1.71 \pm 0.74$ & $2.38 \pm 1.97$ & -1.885 & 0.075 \\
\hline Contralateral lens $D_{\text {mean }}$ & $1.34 \pm 0.55$ & $1.84 \pm 1.69$ & -1.585 & 0.129 \\
\hline Ipsilateral optic nerve $D_{\max }$ & $12.54 \pm 17.85$ & $12.32 \pm 13.38$ & 0.156 & 0.877 \\
\hline Ipsilateral optic nerve $D_{\text {mean }}$ & $8.55 \pm 11.99$ & $8.57 \pm 9.40$ & -0.014 & 0.989 \\
\hline $\begin{array}{l}\text { Contralateral optic } \\
\text { nerve } D_{\max }\end{array}$ & $7.55 \pm 8.94$ & $7.02 \pm 7.68$ & 1.025 & 0.318 \\
\hline $\begin{array}{l}\text { Contralateral optic } \\
\text { nerve } D_{\text {mean }}\end{array}$ & $5.14 \pm 5.92$ & $4.59 \pm 4.76$ & 1.364 & 0.188 \\
\hline Optic chiasm $D_{\max }$ & $12.53 \pm 15.50$ & $12.76 \pm 13.53$ & -0.241 & 0.812 \\
\hline Optic chiasm $D_{\text {mean }}$ & $9.22 \pm 11.64$ & $8.28 \pm 8.70$ & 1.060 & 0.302 \\
\hline Brainstem $D_{\max }$ & $14.78 \pm 14.77$ & $14.04 \pm 11.62$ & 0.523 & 0.607 \\
\hline Brainstem $D_{\text {mean }}$ & $7.09 \pm 8.31$ & $6.52 \pm 6.99$ & 0.711 & 0.486 \\
\hline
\end{tabular}


Table 3 Comparion of radiation dose between 3DCRT and IMRT

\begin{tabular}{|c|c|c|c|c|}
\hline & 3DCRT (Gy) & IMRT (Gy) & $t$ & $p$ \\
\hline Ipsilateral PMC D $D_{\max }$ & $46.50 \pm 8.65$ & $46.54 \pm 7.77$ & -0.050 & 0.960 \\
\hline Ipsilateral PMC D $D_{\text {mean }}$ & $28.45 \pm 7.78$ & $27.67 \pm 8.06$ & 1.429 & 0.169 \\
\hline Contralateral PMC D $\max$ & $24.86 \pm 9.89$ & $21.40 \pm 10.94$ & 1.542 & 0.140 \\
\hline Contralateral PMC D mean & $14.73 \pm 6.02$ & $14.11 \pm 7.57$ & 0.390 & 0.701 \\
\hline Ipsilateral CST D $\max$ & $51.26 \pm 4.24$ & $50.61 \pm 4.72$ & 1.801 & 0.088 \\
\hline Ipsilateral CST D mean & $36.51 \pm 6.63$ & $36.06 \pm 7.57$ & 0.893 & 0.383 \\
\hline Contralateral CST $D_{\max }$ & $35.64 \pm 10.15$ & $36.11 \pm 10.05$ & -0.859 & 0.401 \\
\hline Contralateral CST D mean & $20.97 \pm 7.43$ & $18.90 \pm 7.45$ & 1.454 & 0.162 \\
\hline
\end{tabular}

(Dmax, Dmean, CI and HI) were analyzed and showed no significant differences between two groups. The Dmax and Dmean to these conventional OARs (e.g., brainstem, optic chiasm, optic nerves, and lenses) showed no significant differences between the two plans. The Dmax to the ipsilateral and contralateral PMC and CST regions was considerably decreased by $28.7 \%, 24.5 \%, 20.2 \%$ and $37.6 \%$, respectively. The Dmean to the ipsilateral and contralateral PMC and CST regions was considerably decreased by $27.8 \%, 30.4 \%, 23.1 \%$ and $33.4 \%$, respectively (Table 6).

\section{Discussion}

Radiation therapy is commonly applied to the brain tumors due to its ability to control cell growth; however, radiation therapy can have detrimental effects on the central nervous system causing neurological complications. The response of cerebral tissue to radiation can lead to the deficits in neural functions $[9,10]$. The extent of neurologic deficit is associated with the location and size of radiation-induced brain injury $[11,12]$. Efforts dedicated to the precise division of brain lesions have been made to reduce the risk of neurological complications caused by the radiation therapy.

Table 4 Comparison of target coverage between IMRT and IMRT_PMC\&CST

\begin{tabular}{lllll}
\hline & IMRT & IMRT_PMC\&CST & $\boldsymbol{t}$ & $\boldsymbol{p}$ \\
\hline PTV1 (50 Gy) & & & & \\
$\mathrm{D}_{\max }(\mathrm{Gy})$ & $64.88 \pm 0.66$ & $65.04 \pm 0.70$ & -0.806 & 0.430 \\
$\mathrm{D}_{\text {mean }}(\mathrm{Gy})$ & $59.91 \pm 0.85$ & $59.85 \pm 0.68$ & 0.633 & 0.534 \\
Cl & $1.071 \pm 0.025$ & $1.073 \pm 0.024$ & -1.077 & 0.295 \\
$\mathrm{HI}$ & $0.213 \pm 0.012$ & $0.209 \pm 0.016$ & 0.911 & 0.374 \\
PTV2 (60 Gy) & & & & \\
$\mathrm{D}_{\max }(\mathrm{Gy})$ & $64.88 \pm 0.66$ & $65.04 \pm 0.70$ & -0.806 & 0.430 \\
$\mathrm{D}_{\operatorname{mean}}$ (Gy) & $62.36 \pm 0.53$ & $62.41 \pm 0.56$ & -0.287 & 0.777 \\
Cl & $1.055 \pm 0.049$ & $1.039 \pm 0.047$ & 1.643 & 0.117 \\
$\mathrm{HI}$ & $0.082 \pm 0.016$ & $0.089 \pm 0.016$ & -1.646 & 0.116 \\
\hline
\end{tabular}

Table 5 Comparison of OAR sparing between IMRT and IMRT_PMC\&CST

\begin{tabular}{lllll}
\hline & IMRT (Gy) & $\begin{array}{l}\text { IMRT_PMC\&CST } \\
(\mathbf{G y})\end{array}$ & $\boldsymbol{t}$ & $\boldsymbol{p}$ \\
\hline Ipsilateral lens $D_{\max }$ & $2.67 \pm 1.67$ & $2.76 \pm 1.64$ & -1.574 & 0.132 \\
Ipsilateral lens $D_{\text {mean }}$ & $1.98 \pm 1.45$ & $2.03 \pm 1.50$ & -1.294 & 0.211 \\
Contralateral lens $D_{\max }$ & $2.38 \pm 1.97$ & $2.43 \pm 1.95$ & -1.063 & 0.126 \\
Contralateral lens & $1.84 \pm 1.69$ & $1.89 \pm 1.72$ & -0.940 & 0.359 \\
$D_{\text {mean }}$ & & & & \\
Ipsilateral optic nerve & $12.32 \pm 13.38$ & $12.44 \pm 13.91$ & -0.599 & 0.556 \\
$D_{\text {max }}$ & & & & \\
Ipsilateral optic nerve & $8.57 \pm 9.40$ & $8.78 \pm 10.08$ & -1.126 & 0.274 \\
$D_{\text {mean }}$ & & & & \\
Contralateral optic & $7.02 \pm 7.68$ & $7.07 \pm 7.71$ & -0.353 & 0.728 \\
nerve $D_{\text {max }}$ & & & -0.729 & 0.475 \\
Contralateral optic & $4.59 \pm 4.76$ & $4.66 \pm 4.95$ & & \\
nerve $D_{\text {mean }}$ & & & 1.488 & 0.153 \\
Optic chiasm $D_{\max }$ & $12.76 \pm 13.53$ & $12.49 \pm 13.20$ & 0.573 & 0.573 \\
Optic chiasm $D_{\text {mean }}$ & $8.28 \pm 8.70$ & $8.20 \pm 8.63$ & 1.260 & 0.223 \\
Brainstem $D_{\max }$ & $14.04 \pm 11.62$ & $13.56 \pm 11.08$ & 0.790 & 0.439 \\
Brainstem $D_{\operatorname{mean}}$ & $6.52 \pm 6.99$ & $6.46 \pm 7.05$ & &
\end{tabular}

The DTI and BOLD-fMRI have recently been used to identify the white-matter pathways and functional structures of the brain. In our previous study, we proposed a clinically feasible protocol of integrating BOLD-fMRI and DTI to optimize the extent of resection involving the cortical motor areas and subcortical white matter tracts in patients with brain gliomas. Those information helped neurosurgeons resected the maximum amount of tumor while still preserving the most critical cortices of the brain, thus resulting in enhanced postoperative quality of life for patients [13]. The incorporation of this information for radiosurgery planning has also been suggested. Liu et al. has reported a novel method to integrate the fMRI brain activation map with treatment planning for stereotactic radiosurgery (SRS). Direct irradiation of the eloquent cortices was avoided by multiple radiation arcs or static radiation beams in SRS planning, and the average

Table 6 Comparion of radiation doses betweeen IMRT and IMRT_PMC\&CST

\begin{tabular}{lllll}
\hline & IMRT (Gy) & $\begin{array}{l}\text { IMRT_PMC\& } \\
\text { CST (Gy) }\end{array}$ & $\boldsymbol{t}$ & $\boldsymbol{p}$ \\
\hline Ipsilateral PMC D $\max$ & $46.54 \pm 7.77$ & $33.20 \pm 11.13$ & 7.304 & $<0.001$ \\
Ipsilateral PMC D mean & $27.67 \pm 8.06$ & $19.99 \pm 8.78$ & 7.150 & $<0.001$ \\
Contralateral PMC D max & $21.40 \pm 10.94$ & $16.16 \pm 9.07$ & 5.250 & $<0.001$ \\
Contralateral PMC D & $14.11 \pm 7.57$ & $9.82 \pm 5.62$ & 5.276 & $<0.001$ \\
Ipsilateral CST D max & $50.61 \pm 4.72$ & $40.37 \pm 6.55$ & 9.233 & $<0.001$ \\
Ipsilateral CST D mean & $36.06 \pm 7.57$ & $27.72 \pm 8.87$ & 8.032 & $<0.001$ \\
Contralateral CST D $D_{\text {max }}$ & $36.11 \pm 10.05$ & $22.52 \pm 10.36$ & 6.959 & $<0.001$ \\
Contralateral CST D mean & $18.90 \pm 7.45$ & $12.59 \pm 5.51$ & 6.362 & $<0.001$ \\
\hline
\end{tabular}


dose reduction to the eloquent cortices was 32\% [14]. In addition, it has been reported that the risk of radiationinduced neuropathy was minimized by the integration of tractography of the brain white matter with DTI into radiation treatment planning of radiosurgery using Gamma Knife [15]. Moreover, Pantelis et al. has demonstrated that critical structures of brain could be marked and spared with the aid of the integration of BOLD-fMRI and DTI into CyberKnife stereotactic radiosurgery [16].

In this study, BOLD-fMRI and DTI were used to localize the bilateral PMCs and CSTs and the information obtained from these two technologies were integrated into radiation treatment planning. The first part of our study (3DCRT versus IMRT) indicated that there was no significant reduction in the dose to bilateral PMCs and CSTs between the 3DCRT and IMRT plans. The critical structures adjacent to the target volume marked as OARs can be better spared during the IMRT planning process due to a steep dose gradient and a high conformity $[17,18]$. The second part of our study (IMRT versus IMRT_ PMC\&CST), has shown that a significant reduction in the dose to bilateral PMCs and CSTs regions can be achieved without compromising the coverage of planning target volume and the limiting dose to these conventional OARs.

Sparing of the bilateral PMCs and CSTs does not represent any significant breakthrough in the treatment of brain tumors, but we have demonstrated that it is feasible to reduce the irradiation of critical structures adjacent to the target volume. The development of the most appropriate IMRT plan for the patient could be achieved by the identification of the important functional structures of the brain tissues proximal to the tumors. Sparing these vital functional structures is important to maintain quality of life, even in those patients with restricted life expectancy. The current study has shown that the DTI examination and MR Spectroscopy are valuable tools to differentiate the postoperative recurrent glioma from the radiation injury for patients with a glioma $[19,20]$.

We are currently investigating paradigms for Broca's and Wernicke's areas (speech center), Broadmann-17 functional structures (visual center) and optic tracts. The application of the fMRI in low grade cases has also been validated.

\section{Conclusions}

In conclusion, integration of BOLD-fMRI and DTI into radiation treatment planning is feasible and beneficial. With the assistance of the above-described techniques, the structures adjacent to suspicious cancerous lesions could be clearly marked as OARs and spared during treatment. However, a wider investigation and the longer-term clinical follow up are required to further validate the effect of the integration of BOLD-fMRI and DTI on sparing normal tissues.

\section{Abbreviations}

BOLD-fMRI: Blood oxygen level dependent functional magnetic resonance imaging; DTI: Diffusion tensor imaging; 3DCRT: Three-dimensional conformal radiation treatment; PMCs: Primary motor cortexes; CSTs: Corticospinal tracts; CT: Computed tomography; OARs: Organs at risk; IMRT: Intensity-modulated radiation therapy; IMRT_PMC\&CST: Intensity-modulated radiotherapy planning with PMC and CST information; WHO: World Health Organization; MRI: Magnetic resonance imaging; SE-EPI: Spin echo-echo planar imaging; ROI: Region of interest; GTV: Gross tumor volume; CTV1: Initial clinical target volume; PTV1: Initial planning target volume; CTV2: Second clinical target volume; PTV2: Second planning target volume; DVHs: Dose volume histograms; Dmax: The maximum dose; Dmean: Mean dose; Cl: Conformity index; HI: Homogeneity index.

\section{Competing interests}

The authors declare that they have no competing interests.

\section{Authors' contributions}

$X D W, H C X, X Y H$ and XXJ conceived the study and draft the manuscript. MLW and XDW performed the statistical analysis and drafted the manuscript. HM, YHG, XSX, YLG and HH participated in the analysis of the treatment of radiotherapy plans and draft this manuscript. YX edited this manuscript. All authors read and approved the final manuscript.

\section{Acknowledgements}

This work was supported by the Natural Science Foundation of China (Grant No. 81260373) and the Natural Science Foundation of Ningxia (Grant No. NZ11269).

\section{Author details}

'Department of Radiology, General Hospital of Ningxia Medical University, Yinchuan, China. ${ }^{2}$ Ningxia Key Laboratory for Cerebrocranial Diseases, Yinchuan, China. ${ }^{3}$ Department of Neurosurgery, General Hospital of Ningxia Medical University, Yinchuan, China. ${ }^{4}$ Department of Radiation Oncology, General Hospital of Ningxia Medical University, Yinchuan, China. ${ }^{5}$ Department of Radiology, Xi'an NO.1 Hospital, Xi'an, China. ${ }^{6}$ Tissue Organ Bank \& Tissue Engineering Centre, General Hospital of Ningxia Medical University, Yinchuan, Ningxia, China. ${ }^{7} T$ issue Repair and Regeneration Program, Institute of Health and Biomedical Innovation, Queensland University of Technology, Kelvin Grove, QLD, Australia.

Received: 28 October 2014 Accepted: 19 February 2015 Published online: 08 March 2015

\section{References}

1. Chang J, Narayana A. Functional MRI for radiotherapy of gliomas. Technol Cancer Res Treat. 2010;9(4):347-58.

2. Bleehen NM, Stenning SP. A Medical Research Council trial of two radiotherapy doses in the treatment of grades 3 and 4 astrocytoma. The Medical Research Council Brain Tumour Working Party. Br J Cancer. 1991;64(4):769-74.

3. Butler JM, Rapp SR, Shaw EG. Managing the cognitive effects of brain tumor radiation therapy. Curr Treat Options Oncol. 2006;7(6):517-23.

4. Roman DD, Sperduto PW. Neuropsychological effects of cranial radiation: current knowledge and future directions. Int J Radiat Oncol Biol Phys. 1995;31(4):983-98.

5. Kamada K, Todo T, Masutani $Y$, Aoki S, Ino K, Takano T, et al. Combined use of tractography-integrated functional neuronavigation and direct fiber simulation. J Neurosurg. 2005;102(4):664-72.

6. Pirotte B, Voordecker P, Neuroschl C, Baleriaux D, Wikler D, Metens T, et al. Combination of functional magnetic resonance imaging-guided neuronavigation and intraoperative cortical brain mapping improves targeting of motor cortex stimulation in neuropathic pain. Neurosurgery. 2008;62(6 Suppl 3):941-56.

7. Feuvret L, Noël G, Mazeron JJ, Bey P. Conformity index: a review. Int J Radiat Oncol Biol Phys. 2006;64(2):333-42.

8. Hodapp N. The ICRU report 83: prescribing, recording and reporting photon-beam intensity-modulated radiation therapy (IMRT). Strahlenther Onkol. 2012;188(1):97-9.

9. Keime-Guibert F, Napolitano M, Delattre JY. Neurological complications of radiotherapy and chemotherapy. J Neurol. 1998;245(11):695-708. 
10. DeAngelis LM, Delattre J-Y, Posner JB. Radiation-induced dementia in patients cured of brain metastases. Neurology. 1989;39(6):789-96.

11. Flickinger JC, Kondziolka D, Lunsford LD, Kassam A, Phuong LK, Liscak R, et al. Development of a model to predict permanent symptomatic postradiosurgery. Int J Radiat Oncol Biol Phys. 2000;46(5):1143-8.

12. Voges J, Treuer H, Sturm V, Büchner C, Lehrke R, Kocher M, et al. Risk analysis of linear accelerator radiosurgery. Int J Radiat Oncol Biol Phys. 1996;36(5):1055-63.

13. Jia XX, Yu Y, Wang XD, Ma H, Zhang QH, Huang XY, et al. fMRI-driven DTT assessment of corticospinal tracts prior to cortex resection. Can J Neurol Sci. 2013;40(4):558-63.

14. Liu WC, Schulder M, Narra V, Kalnin AJ, Cathcart C, Jacobs A, et al. Functional magnetic resonance imaging aided radiation treatment planning. Med Phys. 2000;27(7):1563-72.

15. Maruyama K, Kamada K, Shin M, Itoh D, Aoki S, Masutani Y, et al. Integration of threedimensional corticospinal tractography into treatment planning for gamma knife surgery. J Neurosurg. 2005;102(4):673-7.

16. Pantelis E, Papadakis N, Verigos K, Stathochristopoulou I, Antypas C, Lekas L, et al. Integration of functional MRl, white matter tractography in stereotactic radiosurgery clinical practice. Int J Radiat Oncol Biol Phys. 2010;78(1):257-67.

17. Moretto F, Rampino M, Munoz F, Ruo Redda MG, Reali A, Balcet V, et al. Conventional 2D (2DRT) and 3D conformal radiotherapy (3DCRT) versus intensity-modulated radiotherapy (IMRT) for nasopharyngeal cancer treatment. Radiol Med. 2014;119(8):634-41.

18. Arbea L, Ramos LI, Martinez-Monge R, Moreno M, Aristu J. Intensitymodulated radiation therapy (IMRT) vs. 3D conformal radiotherapy (3DCRT) in locally advanced rectal cancer (LARC): dosimetric comparison and clinical implications. Radiat Oncol. 2010;5:17.

19. Xu JL, Li YL, Lian JM, Dou SW, Yan FS, Wu H, et al. Distinction between postoperative recurrent glioma and radiation injury using MR diffusion tensor imaging. Neuroradiology. 2010;52(12):1193-9.

20. Rabinov JD, Lee PL, Barker FG, Louis DN, Harsh GR, Cosgrove GR, et al. In vivo 3-T MR spectroscopy in the distinction of recurrent glioma versus radiation effects: initial experience. Radiology. 2002;225(3):871-9.

\section{Submit your next manuscript to BioMed Central and take full advantage of:}

- Convenient online submission

- Thorough peer review

- No space constraints or color figure charges

- Immediate publication on acceptance

- Inclusion in PubMed, CAS, Scopus and Google Scholar

- Research which is freely available for redistribution 